\title{
Game mechanics application in the optimization and automation framework of personnel management processes
}

\author{
Igor Kotsyuba ${ }^{1, *}$, Konstantin Loginov ${ }^{1}$, and Aleksey Shikov ${ }^{2}$ \\ ${ }^{1}$ ITMO University, Kronverksky Avenue, 49, 197101 Saint Petersburg, Russia \\ ${ }^{2}$ Russian Academy of National Economy and Public Administration under the President of the Russian \\ Federation (North-West Institute of Management - a branch of RANEPA), Sredny prospect V.O., \\ 57/43, 199178 Saint Petersburg, Russia
}

\begin{abstract}
The article examines the processes of applying game mechanics to optimize and automate the management and development of company personnel. This allows one to free up resources for solving tasks that require high qualifications, enhances the quality of work performed by reducing the factor of human errors in monotonous actions, and also ensures the engagement and motivation of employees. It is concluded that solving problems of optimization and automation of personnel management processes is an integral part of the competitive companies' activities. Employees' motivation and engagement in these processes increase their efficiency and loyalty to the company. The use of game mechanics in the implementation of corporate HR management processes increases the motivation and engagement of employees, and, as a result, greatly enhances their efficiency.
\end{abstract}

\section{Introduction}

An indisputable the modern reality fact of enterprises' existence and competitiveness is the presence of highly qualified motivated and involved personnel. For this reason, it is fundamental to have well-built, highly organized business processes for management and personnel development. Like any business processes that are scalable for a large number of people, HR processes involve the prevailing amount of routine work and have a number of requirements and limitations. Optimization and automation of these processes allows freeing up resources for solving tasks that require high qualifications, improves the quality of work performed by reducing the factor of human errors in monotonous actions, and also involves and motivates personnel.

Motivation is viewed as a driver that encourages efforts to achieve goals and is a significant factor in the effective activities' implementation.[1] Moreover, motivation directly affects the time and volume of these very efforts that a person is willing to spend on solving problems [2]. On the other hand, motivation can be considered as a result of activities,

\footnotetext{
* Corresponding author: igor.kotciuba@gmail.com
} 
which is why it is so important to carefully consider all the processes in which employees are involved.

Recently, researchers in the field of training implementation, including corporate training, have been paying more and more attention to the introduction of gamification as a tool to increase the engagement and motivation of students in training [3, 4, 5, 6, 7]. It is believed that the training implementation using gamified services and platforms has the potential to increase the training efficiency, based on the following aspects: providing students with the opportunity to overcome difficulties and challenges; the ability to aggregate knowledge obtained in various ways and from different sources, as well as use it to make prudent decisions; forecast results based on decisions made; encourage students for their activities; provide interactive feedback; stimulate gradual systematic training at a calm comfortable pace $[8,9,10,11]$.

\section{Materials and methods}

In general, gamification is understood as "the use of video game elements in non-gaming systems to improve user experience and user engagement" [12]. The game mechanics are that kind of game elements. Game mechanics mean certain scenario elements that describe how to change the user's game state. In other words, game mechanics are a set of actions that a user can perform, as well as a set of rules and restrictions.

The following main areas of gamification application can be distinguished within the framework of the personnel management processes automation:

1. At the stage of candidates' selection.

2. As part of the adaptation process.

3. In order to support the personnel development process.

The candidates' selection process is one of the most important in the company, determining the staffing of the organization. The main task of a hiring specialist is to find the most competent candidates for a specific position, as well as their engagement in the company.

Let there be $\mathrm{N}$ number of vacancies and $\mathrm{M}$ employees. Each i-th employee can perform each $\mathrm{j}$-th task with a certain efficiency coefficient $u_{i j}$, the value of which can be increased by using one or another set of game mechanics. Then the task of selecting candidates can be formalized as follows:

$$
\mathrm{f}(\mathrm{x})=\sum_{i=1}^{m} \sum_{y=1}^{n} u_{i j} x_{i j} \rightarrow \max
$$

where $u_{i j}$ is the coefficient of utility of the $\mathrm{i}$-th worker for the $\mathrm{j}$-th job role;

$$
\begin{gathered}
D=\left\{x_{i j} \in \mathbb{R}^{m * n} \mid \sum_{i=1}^{m} x_{i j} \leq a_{j}, \forall j=\overline{1, n}\right. \\
\left.\sum_{y=1}^{n} x_{i j} \leq 1 \forall i=\overline{1, m}\right\}
\end{gathered}
$$

Each employee can take no more than 1 role in the team.

$$
x_{i j}=\left\{\begin{array}{c}
1, \text { if } i-\text { th сотеmployee is in the } j-\text { th role }, \\
0, \text { in case he is not. }
\end{array}\right.
$$

One of the key existing issues in personnel management is the staff turnover problem. Relatively high employee turnover rates negatively affect jobseekers' loyalty to the company, which leads to a decrease in the likelihood of hiring highly qualified professionals. This problem is most acutely perceived by innovative companies and enterprises, for which the time spent on the adaptation process of new employees should be reduced to a minimum, and 
the adaptation process itself should be the most effective. Automation of the personnel adaptation process through the development and implementation of an information system for personnel management and development can significantly reduce the time spent by experienced employees on getting up to speed.

The task of implementing the employees' adaptation can be formulated in terms of the optimization problem (4):

$$
\begin{gathered}
f(x)=\sum_{i=1}^{m} e f f_{i} \cdot x_{i} \rightarrow \max \\
D=\left\{x \in R^{m} \mid x_{i} \in[0,1] \forall i=\overline{1, m}\right. \\
\sum_{i=1}^{m} t(e d u)_{i} \cdot x_{i} \leq T(\text { edu }), \\
\left.\sum_{i=1}^{m} t(\text { adapt })_{i} \cdot x_{i} \leq T(\text { adapt })\right\}
\end{gathered}
$$

where eff is the training efficiency of the $\mathrm{i}$-th employee, $\mathrm{t}(\mathrm{edu})$ is the training time of the $\mathrm{i}$-th employee, $\mathrm{t}$ (adapt) is the adaptation time of the $\mathrm{i}$-th employee, $\mathrm{T}$ (edu) is the total time allocated for training, T (adapt ) - the general fund of time allocated for adaptation. X(i) - a plurality of trained / adaptable employees

Along with the above-mentioned tasks of personnel management, the issue of managing employees' development and training is highlighted. Ensuring the development and continuous maintenance of proficiency level in the competencies necessary for the production process for innovative enterprises is one of the most important conditions for competitiveness and market leadership.

One of the main approaches to organizing training is to focus on the employee competency matrix. The competence matrix is understood as a list of competencies that are valuable for the company and help in the implementation of its tasks. It covers various knowledge, skills and abilities and is organized in the form of competency profiles - sets of competencies and knowledge levels of these competencies necessary for the successful completion of specific tasks. The competence matrix is shown in table 1.

Table 1. Matrix of competencies

\begin{tabular}{|c|c|c|c|c|}
\hline & $\mathrm{c}_{1}$ & $\mathrm{c}_{2}$ & $\ldots$ & $\mathrm{c}_{p}$ \\
\hline$k_{1}$ & & & & \\
\hline$k_{2}$ & & & & \\
\hline$\ldots$ & & & & \\
\hline$k_{r}$ & & & & \\
\hline
\end{tabular}

where $\mathrm{c}_{1}, \mathrm{c}_{2}, \ldots, \mathrm{c}_{p}-\mathrm{a}$ set of competencies corresponding to a specific profile;

$k_{1}, k_{2}, \ldots, k_{r}$ - level of competencies' possession.

Implementation of the concept of the current matrix of employee competencies and the target one allows one to select the most suitable candidates for a vacancy, build training paths for the purpose of vertical or horizontal career growth of employees, track its progress, and form a base of interchangeable personnel. The calculation of deviations of the current competency matrix from the target can be calculated as:

$$
\left\{\begin{array}{c}
k_{1 \text { goal }}-k_{1 \text { cur }} \leq \delta_{1}, \\
k_{2 \text { goal }}-k_{2 c u r} \leq \delta_{2}, \\
\ldots \\
k_{\text {pgoal }}-k_{\text {pcur }} \leq \delta_{p}
\end{array}\right.
$$


where $k_{\text {pgoal }}$ is the target value of the proficiency level in the p-th competence, $k_{\text {pcur }}$ is the current value of the level of proficiency in the $\mathrm{p}$-th competence, $\delta_{p}$ is the permissible deviation of the current level of proficiency in the $\mathrm{p}$-th competence from the target. Thus, the vector will act as a criterion for achieving the target competency profile:

$$
\left(\begin{array}{c}
\delta_{1} \\
\delta_{2,} \\
\ddot{\delta_{p}}
\end{array}\right)
$$

However, the process of training implementation and increasing the competence of personnel must meet a number of requirements: minimizing the time spent on training; minimization of the working hours of the expert teacher; ensuring the maximum efficiency of the training provided. Then this problem can be represented as a multiobjective optimization problem:

$$
\begin{gathered}
f_{1}(x)=\sum_{i=1}^{m} \text { eff }_{i} \cdot x_{i} \rightarrow \max \\
f_{2}(x)=\sum_{i=1}^{m} t(\exp )_{i} \cdot x_{i} \rightarrow \min \\
f_{1}(x)>f_{2}(x) \\
D=\left\{x \in R^{m} \mid x_{i} \in[0,1] \forall i=\overline{1, m}\right. \\
\left.\sum_{i=1}^{m} t(\text { edu })_{i} \cdot x_{i} \leq T(\text { edu })\right\} \\
x_{i}=\left\{\begin{array}{c}
1, \text { if } i-\text { th if applied game mechanics } \\
0, \text { otherwise }
\end{array}\right.
\end{gathered}
$$

where eff is the effectiveness of training using the $\mathrm{i}$-th game mechanics, $t(\mathrm{edu})$ is the training time using the $\mathrm{i}$-th game mechanics, $\mathrm{t}(\exp )$ is the time of the expert's work using the i-th game mechanics. X(i) is the plurality of all game mechanics.

During the operation of the corporate training system, it becomes crucial to assess the effectiveness of the developed system. There are various approaches, that take into account different metrics and indicators, used to assess the effectiveness [13-15]. The most common model for assessing the effectiveness of the corporate training courses implementation is the Kirpatrick's four-level model [16].

The task of conducting corporate training is to ensure its maximum efficiency, while minimizing training costs and time. Then, this kind of problem can be formulated in the form of a multi-criteria optimization problem:

$$
\begin{aligned}
& f_{1}(x)=\sum_{i=1}^{m} \text { firstLev }_{i} \cdot x_{i} \rightarrow \max \\
& f_{2}(x)=\sum_{i=1}^{m} \operatorname{secondLev~}_{i} \cdot x_{i} \rightarrow \max \\
& f_{3}(x)=\sum_{i=1}^{m} \text { thirdLev }_{i} \cdot x_{i} \rightarrow \max \\
& f_{4}(x)=\sum_{i=1}^{m} \text { fourthLev }_{i} \cdot x_{i} \rightarrow \max \\
& D=\left\{x \in R^{m} \mid x_{i} \in[0,1] \forall i=\overline{1, m}\right.
\end{aligned}
$$




$$
\begin{gathered}
\sum_{\substack{i=1 \\
\sum_{i=1}^{m}}}^{m}(e d u)_{i} \cdot x_{i} \leq T(e d u) \\
\left.\cdot x_{i} \leq \operatorname{CoS} T\right\},
\end{gathered}
$$

where $\mathrm{x}_{-} \mathrm{i}$ is the $\mathrm{i}$-th game mechanics from the set $\mathrm{X}, \mathrm{t}(\mathrm{edu})$ is the training time using the i-th game mechanics; T(edu) - general time fund allocated for training; COST - general cost fund, firstLev - fourthLev - achievement indicators of the corresponding four levels according to Kirpatrick. The result of solving this optimization problem is the vector of game mechanics used, which makes it possible to maximize the efficiency of corporate training, taking into account the existing restrictions on the cost of training time.

\section{Results and discussion}

As part of a study to assess the effectiveness of the gamification introduction in the process of personnel corporate training, the authors of this article set up an experiment consisting in passing the developed electronic training course "Agile Methodology", presented in several modifications: with the use of a different set of game mechanics and without them. This course was intended for employees of an innovative software development company. The experiment involved 32 people aged 22 to 34 years. When applying for this course, all participants were randomly divided into three control groups for three types of e-learning courses, depending on the type and number of game mechanics used.

The e-learning course was implemented in three versions:

1. Without using game mechanics (course type 1);

2. Using the mechanics of reward / punishment, the mechanics of competition, the mechanics of recall (course type 2);

3. Using the mechanics of reward / punishment, mechanics of competition, mechanics of reminder and mechanics of prompts (course type 3);

The training course was divided into modules. Two types of deadlines were used as time constraints for the delivery of the final tasks in the module: soft and hard. The soft deadline assumed a decrease in points for completed tasks by $5 \%$ of the points scored for each day of delay, thus implementing the punishment mechanics. The tough deadline meant that there was no way to evaluate assignments if it was violated.

Data analysis obtained on the results of passing the e-learning course showed that the values of the average time for completing course tasks in the case of using game mechanics turned out to be $25 \%-29 \%$ less (depending on the type of course) the time for completing tasks in the absence of gamification elements (Fig.1). This fact is explained by the implementation of the impact of the game mechanics of reward / punishment and competition, which stimulate the students' desire to get the maximum number of points, while observing the existing deadlines.

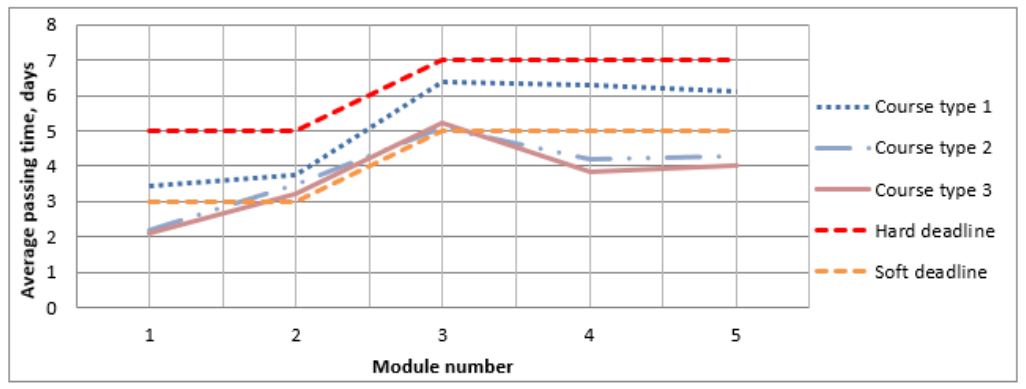

Fig. 1. Dependence completing time of modules tasks on the course type 
We can also conclude that the use of game mechanics of reward / punishment and competition, which gives the possibility of obtaining more points in case of meeting the deadlines for completing the course tasks, contributed to earlier completion of tasks and adherence to deadlines, which ensured almost halve the number of strict deadlines violations.

The effectiveness of the application of the hint game mechanics is also confirmed in the course of the data analysis. The number of correct answers on the second attempt in the case of using the game mechanic of the hint turned out to be $11 \%$ higher than in the case without it, and on the third - by $16 \%$. This fact proves the effectiveness of the application of the hint game mechanics in the framework of the electronic training courses implementation.

Also, the retention rate was chosen as the basic criterion for assessing the effectiveness of the application of the developed solutions. This metric reflects information about what percentage of the initially registered users interacted with the system on day $\mathrm{N}$. The classic calculation of the metric is carried out as follows:

$$
\operatorname{ret}_{N}=\frac{k_{N}}{p_{N}} * 100 \%
$$

where $\operatorname{ret}_{N}$ - day retention rate $\mathrm{N}$;

$k_{N}$ - the number of users logged in per day $\mathbf{N}$;

$p_{N}$ - the number of users who started using the system $\mathrm{N}$ days ago.

In the case of taking courses with the use of gamification elements, on average, the value of the N-day retention rate is higher than in the case of passing the course without introducing game mechanics (Fig. 2). The value of the standard deviation of the retention rate for the period excluding the first and the last two days was $38 \%$ of the average value of this metric in the case of completing the training course without the use of game mechanics, and in the case of their application - $10-13 \%$.

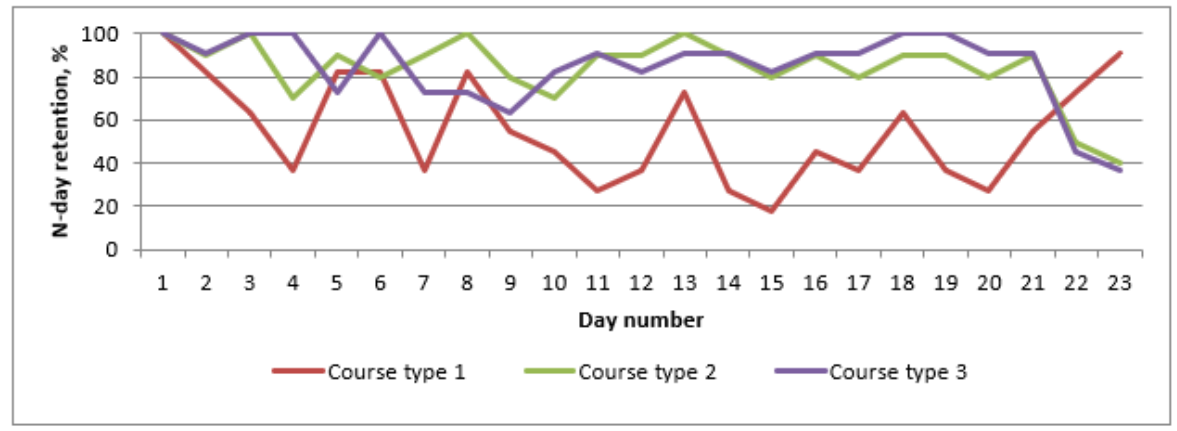

Fig 3. Graph of changes in the N-day retention rate for three types of courses

This fact truthfully indicates that the use of the game mechanics of rewards / punishments contributes to the constant systematic work on the passage of the training course.

\section{Conclusions}

Evaluating the effectiveness according to Kirpatrick's model also showed that all four levels of the model were successfully achieved. The fact that the value of the average upload time of software releases was reduced by $7 \%$, the rate of the number of urgent fixes (hotfixes) made after the release of releases decreased by $17 \%$ indicates the achievement of the fourth level of the model, namely, an improvement in business performance as a result of training.

Thus, it can be concluded that solving the problems of optimization and automation of personnel management processes are an integral part of the competitive companies' 
activities. Motivation and engagement of employees in these processes increase their efficiency, staff loyalty to the company, and create a positive brand image. The use of game mechanics within the framework of the implementation of corporate HR management processes allows to increase the motivation and engagement of employees, and, as a consequence, increase their efficiency.

\section{References}

1. W. Harlen, In J. Gardner (Ed.), Assessment and Learning, 61 (2006)

2. J.D. Bransford, A.L. Brown, R.R. Cocking, How people learn (2004).

3. S. Tobias, J.D. Fletcher (Eds.), Computer games and instruction (2011)

4. M.S.Y. Jong, Curriculum Journal, 26 (2), 249 (2015)

5. M. Kebritchi and A. Hirum, Computers \& Education, 51 (4), 1729 (2008)

6. S. Erhel and E. Jamet, Computers \& Education, 67, 156 (2013)

7. J.L. Plass, B.D. Homer and C.K. Kinzer, Educational Psychologist, 50 (4) , 258 (2015)

8. C. Bouras, V. Igglesis, V. Kapoulas, I. Misedakis, O. Dziabenko, A. Koubek, Journal of Intelligent Games and Simulation, 3 (2), 70 (2004)

9. M.I. Cicchino, Interdisciplinary Journal of Problem-based Learning, 9 (2) (2015).

10. J. McCall, The History Teacher, 46 (1), 9 (2012)

11. B. Kim, H. Park and Y. Baek, Computers \& Education, 52 (4), 800 (2009)

12. S. Deterding, D. Dixon, R. Khaled, L. Nacke, From game design elements to gamefulness: Defining gamification 15th international academic MindTrek conference (2011)

13. J.J. Phillips, Handbook of Training Evaluation and Measurement Methods (1997)

14. A. Adelsberg, E. Trolley, Running Training Like a Business The Forum Corporation (1999)

15. T. Waddington, B. Aaron, R. Sheldrick, Guerilla Evaluation: Adapting to the Terrain and Situaion. Instructional Desing in the Real World (2004)

16. D. Kirkpatrick, Training and Development, 50, 54 (1996) 